\title{
CORRECTION
}

\section{Correction to: National burden of pediatric abusive injuries: patterns vary by age}

\author{
Carlos Theodore Huerta ${ }^{1}$ (C) Eduardo A. Perez ${ }^{1} \cdot$ Hallie Quiroz ${ }^{1} \cdot$ Kirby Quinn $^{1} \cdot$ Chad M. Thorson $^{1}$. \\ Anthony R. Hogan ${ }^{1} \cdot$ Ann-Christina Brady ${ }^{1}$ Juan E. Sola ${ }^{1}$
}

Published online: 22 January 2022

(c) Springer-Verlag GmbH Germany, part of Springer Nature 2022

\section{Correction to: Pediatric Surgery International https://doi.org/10.1007/s00383-022-05062-w}

In Table 4 of this article, under column " $13+$ years $n(\%)$ " and row "Genitourinary" with a value of "10 (0.2)" which should be changed to a superscript " $b$ " similar to the other censored values in the table. The corrected Table 4 is given here.

The original article has been corrected.

Publisher's Note Springer Nature remains neutral with regard to jurisdictional claims in published maps and institutional affiliations.

The original article can be found online at https://doi.org/10.1007/ s00383-022-05062-w.

Carlos Theodore Huerta

cth62@med.miami.edu

1 DeWitt Daughtry Family Department of Surgery, University of Miami Miller School of Medicine, 1800 NW 10th Ave, Miami, FL 33136, USA 
Table 4 Age-related injury patterns among pediatric abuse victims

\begin{tabular}{lllllll}
\hline Injury type & $<1$ year & $\begin{array}{l}1-4 \text { years } \\
n(\%)\end{array}$ & $\begin{array}{l}5-8 \text { years } \\
n(\%)\end{array}$ & $\begin{array}{l}9-12 \text { years } \\
n(\%)\end{array}$ & $\begin{array}{l}13+\text { years } \\
n(\%)\end{array}$ & $p$ value $^{\mathrm{a}}$ \\
\hline Burns & $32(0.2)$ & $55(1)$ & $\mathrm{b}(<1)$ & $0(0)$ & $0(0)$ & $\mathbf{0 . 0 0 1}$ \\
Facial trauma & $561(3)$ & $614(6)$ & $97(3)$ & $97(3)$ & $183(3)$ & $\mathbf{0 . 0 0 1}$ \\
Fractures & $7293(43)$ & $2404(26)$ & $157(5)$ & $68(2)$ & $167(3)$ & $\mathbf{0 . 0 0 1}$ \\
Gastrointestinal & $92(0.5)$ & $367(4)$ & $47(2)$ & $23(1)$ & $\mathrm{b}(<1)$ & $\mathbf{0 . 0 0 1}$ \\
Genitourinary & $30(0.2)$ & $60(1)$ & $\mathrm{b}(<1)$ & $12(0.4)$ & $\mathrm{b}(<1)$ & $\mathbf{0 . 0 0 1}$ \\
Intrathoracic & $373(2)$ & $191(2)$ & $44(1)$ & $22(1)$ & $22(0.4)$ & $\mathbf{0 . 0 0 1}$ \\
Liver & $291(2)$ & $357(3)$ & $12(0.4)$ & $\mathrm{b}(<1)$ & $15(0.3)$ & $\mathbf{0 . 0 0 1}$ \\
Nerve & $75(0.4)$ & $45(0.4)$ & $\mathrm{b}(<1)$ & $0(0)$ & $\mathrm{b}(<1)$ & $\mathbf{0 . 0 0 1}$ \\
Pancreas & $27(0.2)$ & $209(2)$ & $54(2)$ & $16(1)$ & $13(0.2)$ & $\mathbf{0 . 0 0 1}$ \\
Spleen & $80(0.5)$ & $75(1)$ & $\mathrm{b}(<1)$ & ${ }^{\mathrm{b}}(<1)$ & $14(0.2)$ & $\mathbf{0 . 0 0 1}$ \\
Multiple trauma & $10,324(61)$ & $5115(49)$ & $635(21)$ & $436(15)$ & $869(15)$ & $\mathbf{0 . 0 0 1}$ \\
Abuse type & & & & & & \\
Abusive head trauma & $4186(25)$ & $1054(10)$ & $179(6)$ & $137(5)$ & $68(1)$ & $\mathbf{0 . 0 0 1}$ \\
Physical & $8711(51)$ & $5440(53)$ & $1230(40)$ & $1020(36)$ & $1806(31)$ & $\mathbf{0 . 0 0 1}$ \\
Sexual & $1209(7)$ & $917(9)$ & $274(9)$ & $265(9)$ & $251(4)$ & $\mathbf{0 . 0 0 1}$ \\
Emotional & $87(1)$ & $120(1)$ & $93(3)$ & $117(4)$ & $313(5)$ & $\mathbf{0 . 0 0 1}$ \\
Neglect & $0(0)$ & $0(0)$ & $0(0)$ & $\mathrm{b}(<1)$ & $31(1)$ & $\mathbf{0 . 0 0 1}$ \\
\hline
\end{tabular}

${ }^{\text {a }}$ Significance set at $P<0.01$ after Bonferroni correction.

Asterisks $\left({ }^{b}\right)$ represent numeric values censored from publication in compliance with the Healthcare Cost and Utilization Project Data Use Agreement. 